تأثير إضافة تركيزين مختلفين من الكروم العضوي في نسب التصافي والتركيب الكيميائي للحم ثلاث سلالات من فروج اللحم

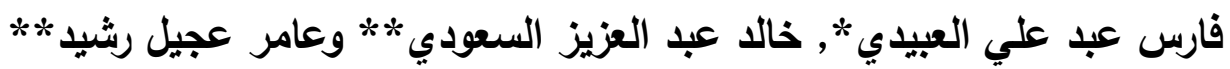

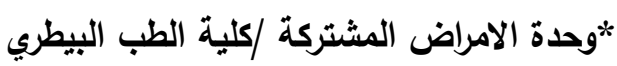

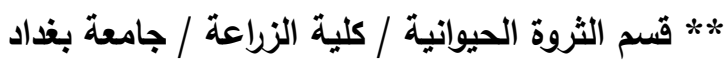

\title{
الخلاصة
}

أجريت الدراسة بهذف تحديد اضافة 0 و 150 و 300 جزء بالبليون من الكروم العضوي في العلف وأثره في نسب التصافي والقطعيات والتركيب الكيميائي للحم ثلاثة سلالات تجارية من فروج اللحم هي لومان وروز وهبرد . وزعت 450 فرخ لحم (150 فرخ من كل سلالة تجارية ) على تسعة معاملات

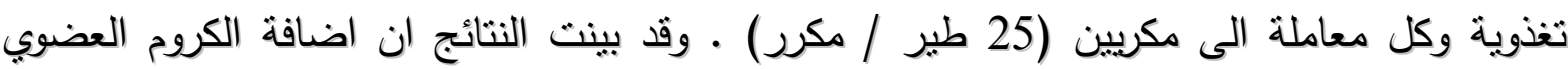

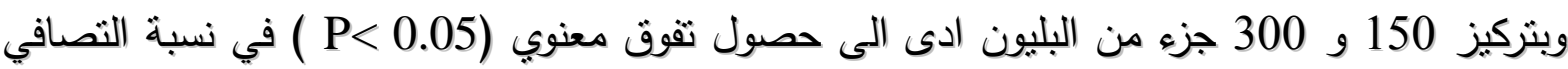

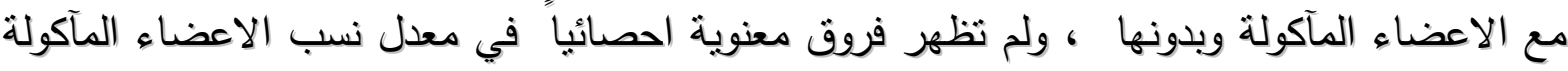

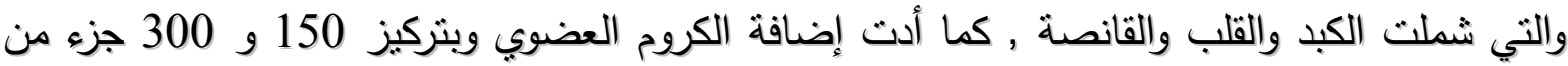
البليون الى زيادة كمية اللحم المنتجة من خلال زيادة نسبة القطيعات الرئيسة معنوياً (

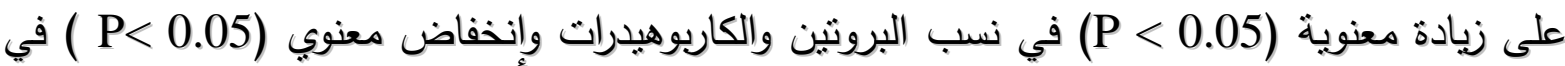
نسب الدهن والرطوبة في لحم القطع الرئيسية . لم يظهر ناثير معنوي للنداخل مابين اضافة الكروم والعوامل الوراثية لسلالات فروج اللحم الثلاثة

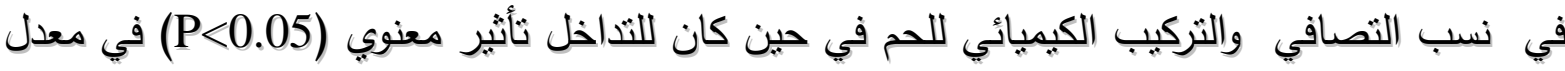

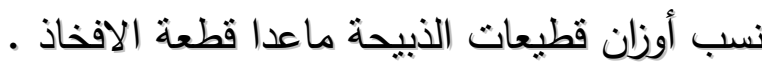

\section{EFFECT OF ADDING TWO DIFFERENT CONCENTRATION OF ORGANIC CHROMIUM ON PERFORMANCE OF THREE BROILER STRAINS}

Faris A. AL-Obaidi*, Khalid A. AL-Soudi** and Amer A. Rashed** *Zoonosis unit / College of Veterinary Medicine/ University of Baghdad

**Dep. Of Animal Resources / College of Agriculture / University of Baghdad

\section{Abstract}

This study was carried out to determined the effect of adding $0,150,200 \mathrm{ppb}$ of organic chromium in feed on dressing percentages and meat composition of three commercial broiler strains (Lohman ,Ross and Hubbard).A total of 450 
day old chicks (150 chicks per strain)were distributed into nine treatments and each treatment

Divided into two replicates ( 25 birds per replicate). The data obtained reveald the following results:

Adding 150 and $300 \mathrm{ppb}$ organic chromium increased significantly $(\mathrm{P}<0.05)$ dressing percentages with or without giblets, and no significant differences appeared in edible organs percentages includes liver, heart and gizzard, also adding 150 or $300 \mathrm{ppb}$ of organic chromium increased meat production through the significant $(\mathrm{P}<0.05)$ increase of primary carcass cuts which were breast and legs, also increased significantly $(\mathrm{P}<0.05)$ protein , carbohydrates and decreased significantly $(\mathrm{P}<0.05)$ lipids, and moisture in these cuts meat.

No significant differences appeared for the interaction between adding chromium and genetic factors for the three broiler strains in dressing percentages and meat composition and the effect was significant $(\mathrm{P}<0.05)$ in carcass cuts except legs percentages .

\section{المقدمة}

يعد الكروم ثلاثي التكافؤ (Cr+3 ) ذو سمية منخفضة جدا وغالبا غير سام وخاصة عند وجوده

بهيئة مركبات عضوية ( Organic compounds) مثل كروم الخميرة ( Yeast - chromium ) , او بهيئة متحدة مع بعض الفيتامينات (1 و 2 ) , وأثنارت الأبحاث الحديثة إلى دوره المهم في المحافظة

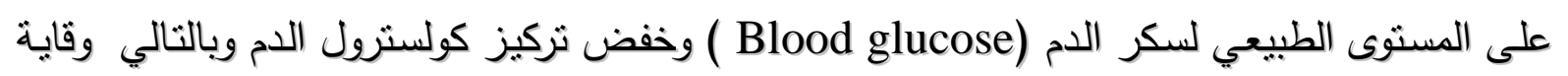

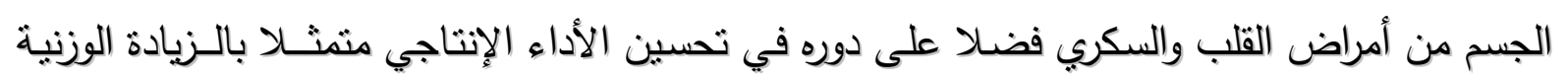

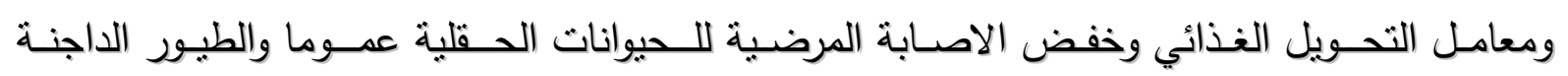
خصوصا (3, 4,5 ) 5 ) ن وجد Ward and Southern (6 ) أن تغذية فروج اللحم على عليقة تحوي نسب من الكروم

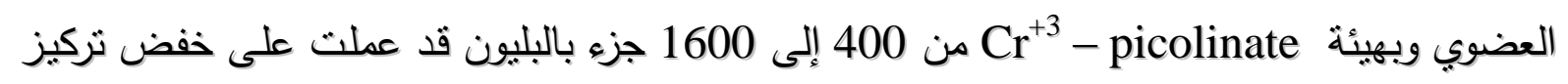

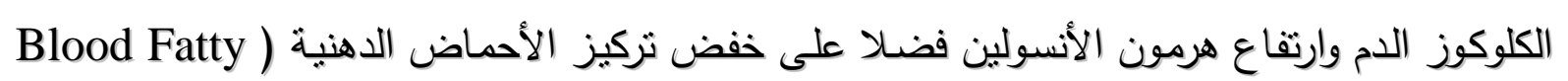

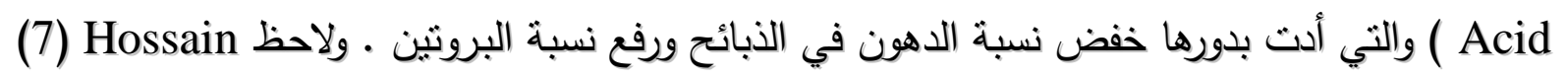

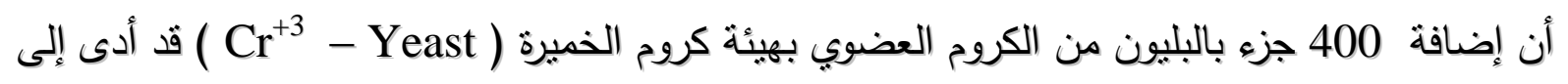

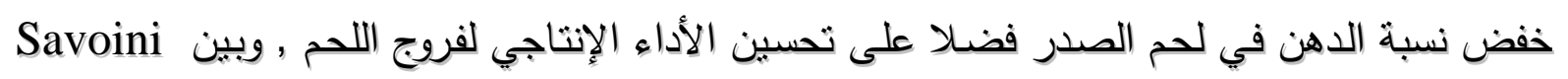

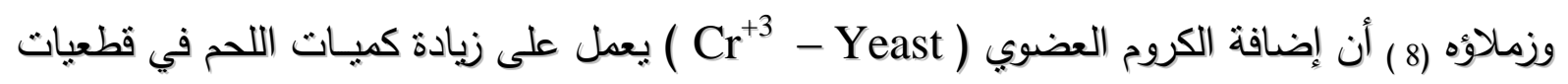

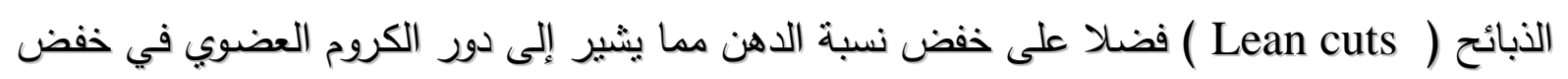
تصنيع الدهون داخل الجسم . 
ولاحظ Hossain (9) أن إضافة 400 جزء بالبليون من كروم الخميرة في علف فروج اللحم عمل على فلى

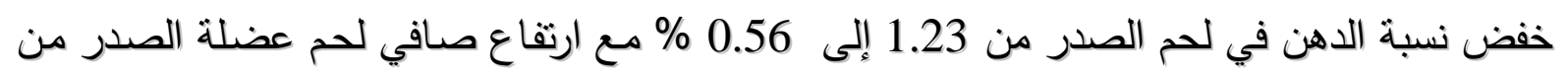
19.3 إلى 20.9 \% فضلا على زيادة وزن قطعة الصدر من 376 إلى 373 إلى 403 غم.

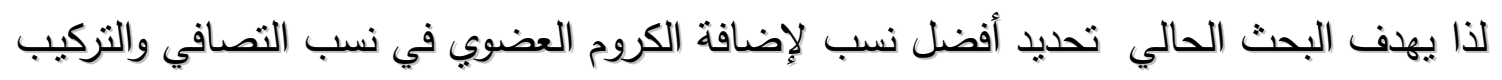

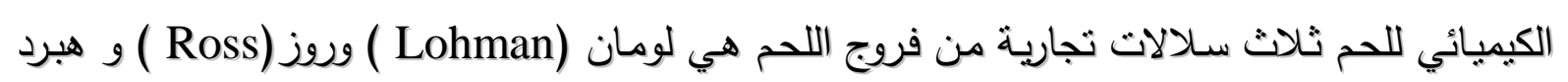
( (Hubbard)

\section{المواد وطرائق العمل}

أجريت هذه التجربة في حقل الطيور الداجنة الثابع لقسم الثروة الحيوانية في كلية الزراعة جامعة بغداد

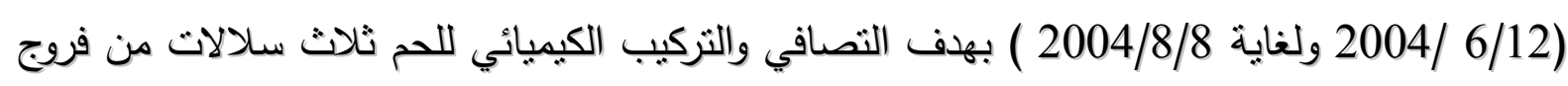

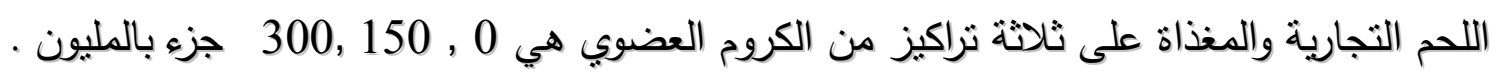
تربية الطيور:

تم توزيع 450 فرخ لحم بعمر يوم واحد (150 فرخ لومان , 150 فرخ روز , 150 فرخ هبرد )

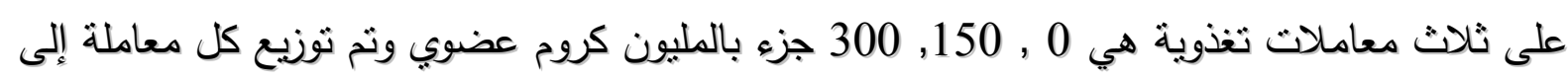

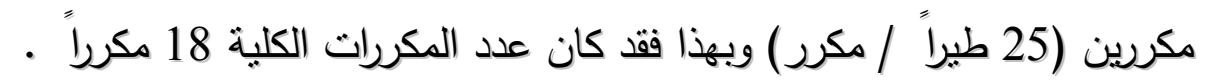
التظذية : غذيت الأفراخ تغذية حرة (Ad Libitum) على عليقة تجهز 21.5 بروثين وطاقة ممثلة 2950 كيلو

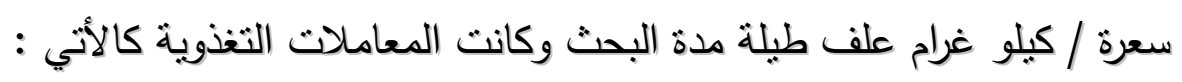

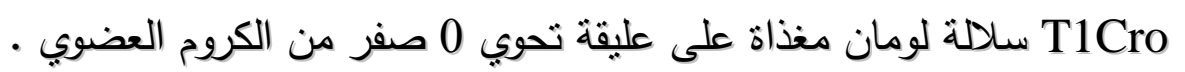
سلالة لومان مغذاة على عليقة تحوي 150 جزء بالمليون من الكروم العضوي. سلالة لومان مغذاة على عليقة تحوي 300 جزء بالمليون من الكروم العضوي.

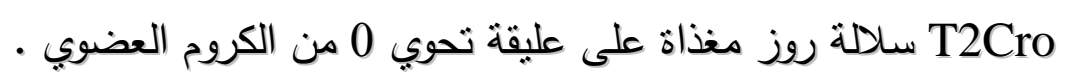
سلالة روز مغذاة على عليقة تحوي 150 جزء بالمليون من الكروم العضوي. سلالة روز مغذاة على عليقة تحوي 300 جزء بالمليون من الكروم العضوي.

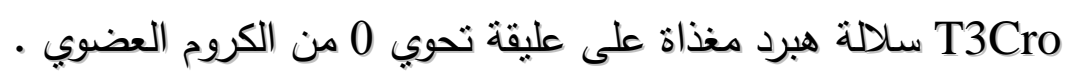
سلالة هبرد مغذاة على عليقة تحوي 150 جزء بالمليون من الكروم العضوي. سلالة هبرد مغذاة على عليقة تحوي 300 جزء بالمليون من الكروم العضوي. 
الكروم العضوي :

تم الحصول على الكروم العضوي نوع كروم الخميرة (Cr-Yeast)والمسمى Biochromium المنتج من شركة Alltech LTD من السوق التجارية والذي يجهز الغرام الواحد منه 100 / ملغم كروم

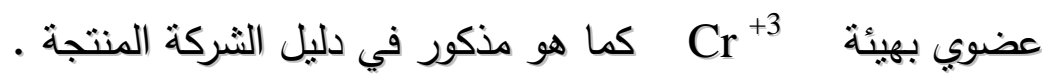

الصفات المدروسة :

عند نهاية مدة التجربة التي استمرت 56 , تم اخذ ثلاثة دجاجات من كل مكرر و جرى ثنجيل

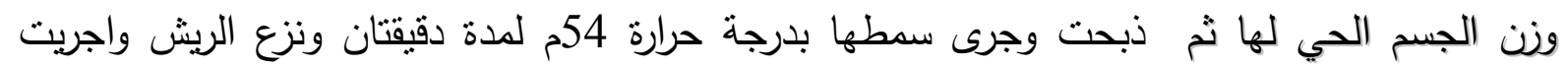

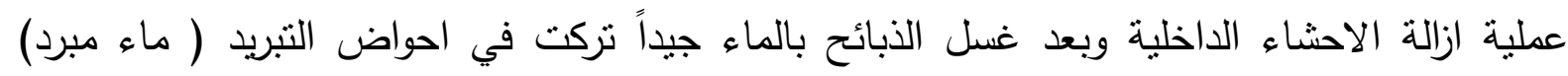

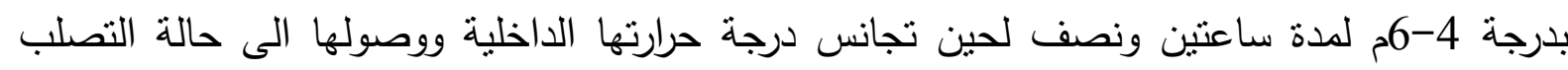
الرمي (Rigor mortis) من اجل وصول الاس الهيدروجيني للحم الى ادنى نقطة له. بعدها جرى دئه

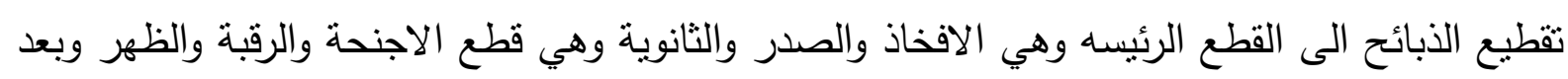

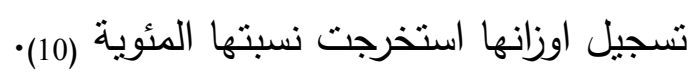

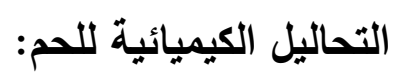
من كل معاملة نم اخذ 3 قطع افخاذ وثلاث قطع صدر ثلاثة انصاف قطع صدر) وجرى فصل اللحم عن العظم وقطعت وفرمت باستخدام مفرمة لحم وعبأ اللحم داخل اكياس من النايلون ثم اغلقت وحفظت أهن

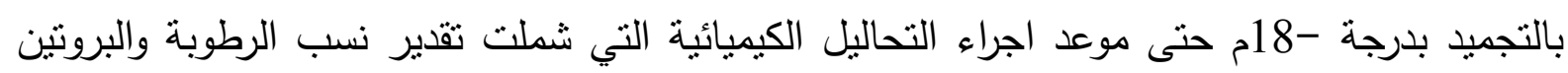

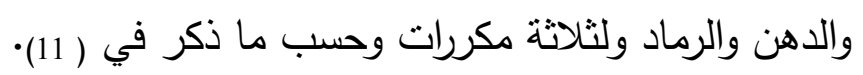

التحليل الإحصائي :

تم تحليل بيانات التجربة حسب تجربة عاملية (Factorial) ضمن التصميم العشوائي الكامل وتم مقارنة الفروقات مابين المتوسطات بإستخدام اختبـار دنكـن متعدد المديات عند مسنوى 0.01 و

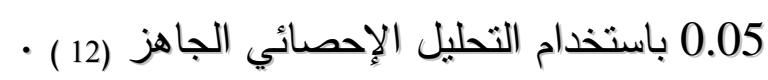
النتائج

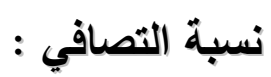

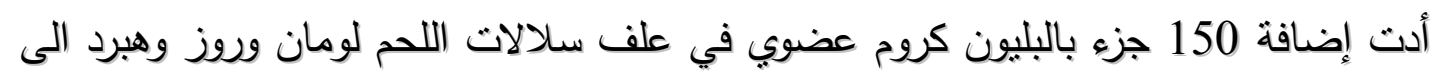

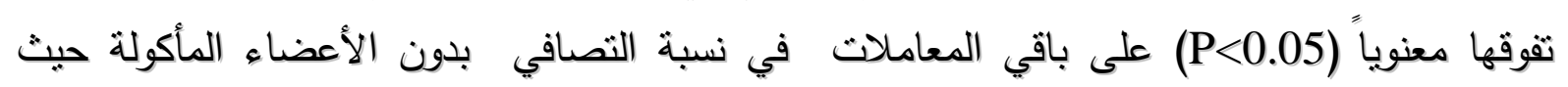

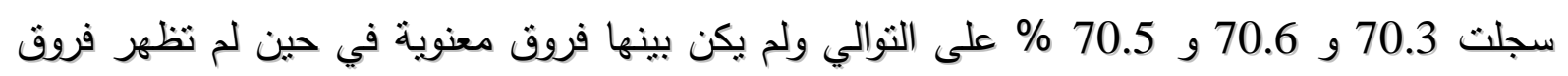

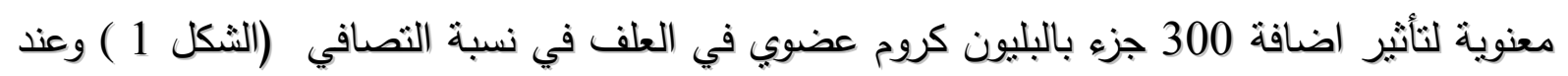

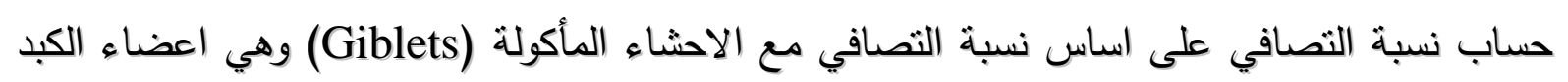


والقلب والقانصة استمر الثقوق المعنوي (P<0.05) لمعاملات سلالات لومان وروز وهبرد المغذاة على علف يحوي 150 جزء بالبليون كروم عضوي على معاملات نفس السلالات المغذاة على علف ولف خالي

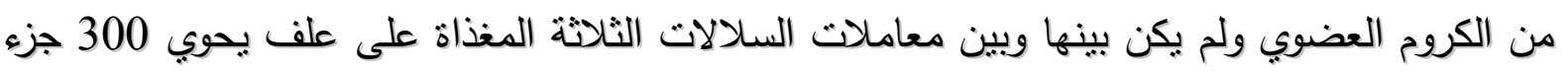

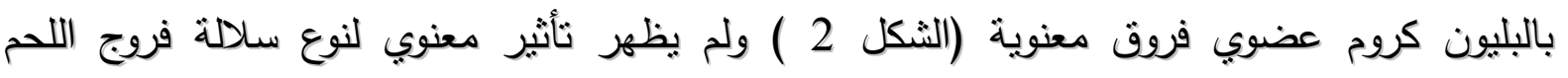

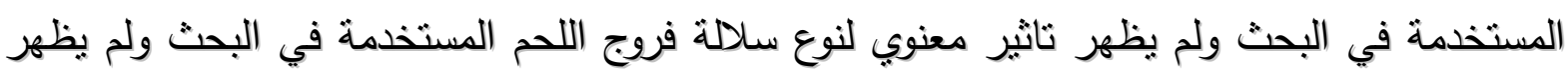

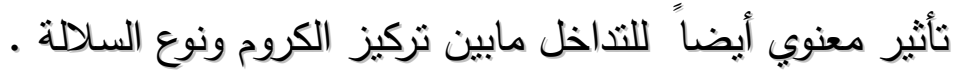

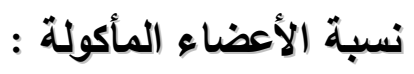

يتضح من الجدول 1 عدم وجود اختلافات معنوية في نسب الأعضاء المأكولة وهي نسبة وزن

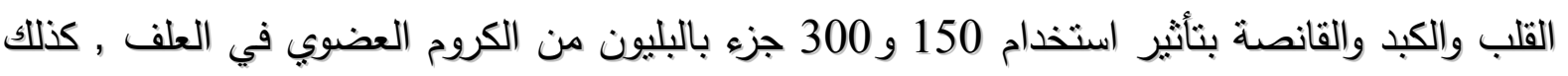

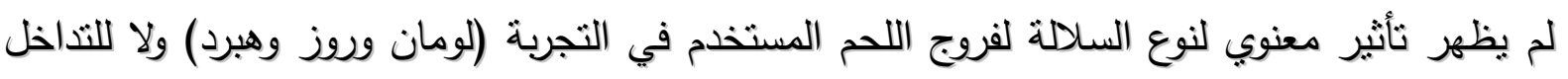

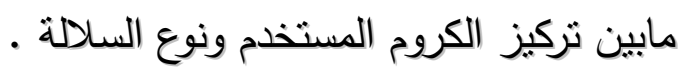

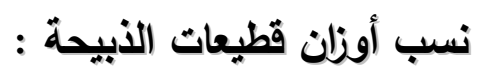
يبين الجدول 2 نأثير اضافة نركيزين مختلفين من الكروم العضوي في نسب اوزان قطبعات الذبيحة

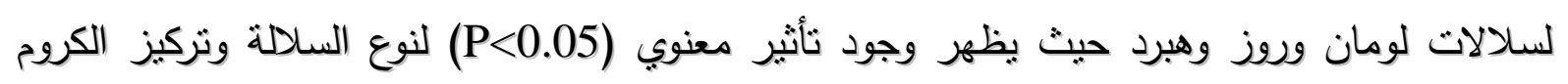
والتداخل مابين السلالة وتركيز الكروم في نسبة قطعة الصدر (Breast \%) إذ إذ سجلت سلالدة لومان

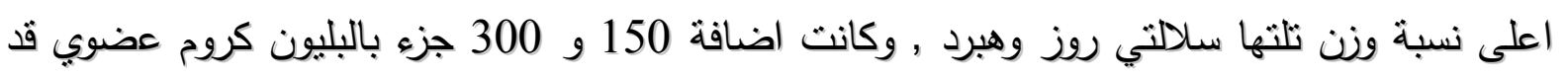

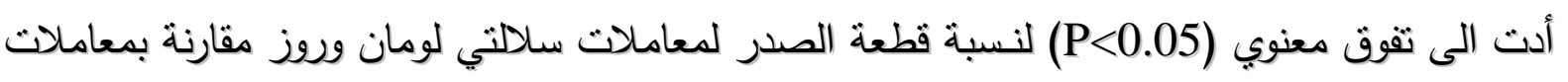

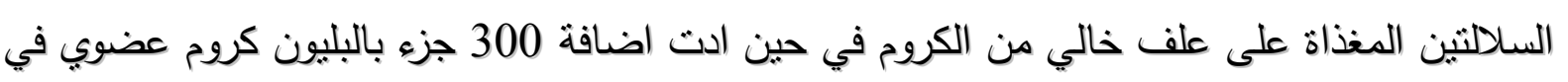

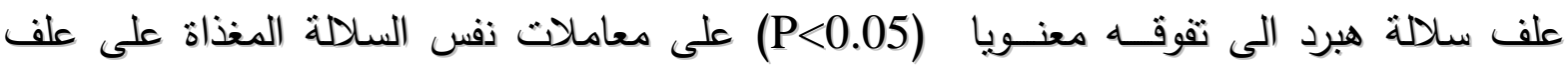

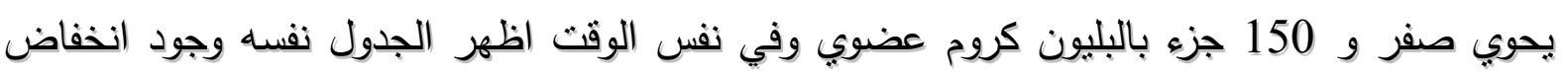

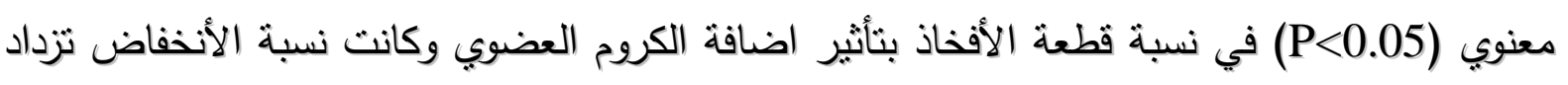

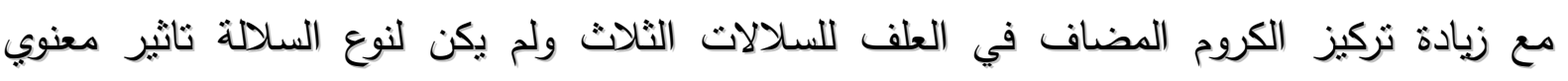

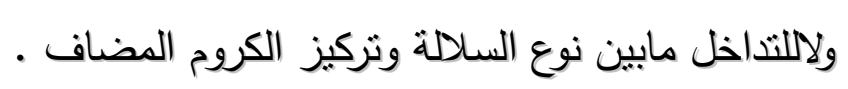

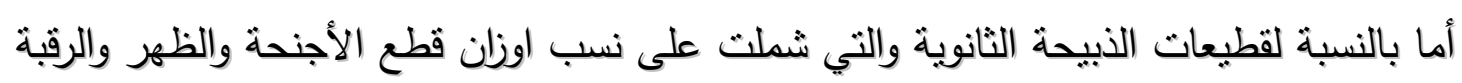

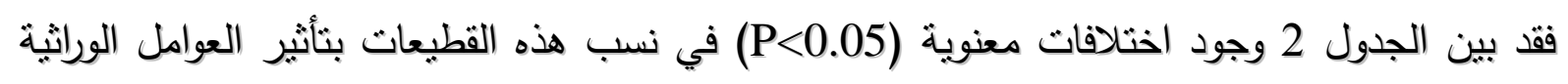

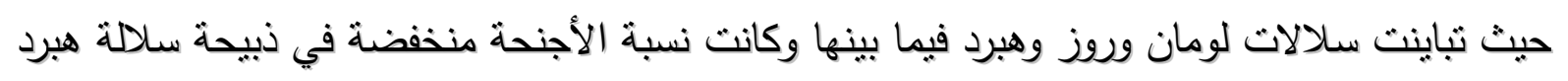

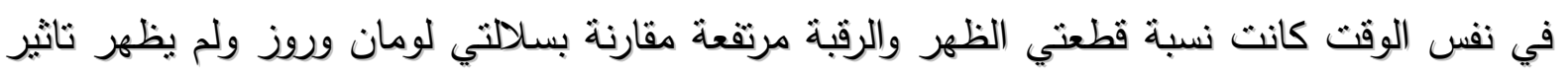


المجلة الطبية البيطرية العراقية، المجلد 30، العدد 2، السنة 2006

للكروم المضاف في نسب هذه القطيعات , إلا ان التداخل مابين تركيز الكروم المضاف ونوع السلالة

$$
\text { كان معنوياً (P<0.05). }
$$

الشكل 1 تأثير اضافة تركيزين مختلفين من الكروم العضوي في نسب التصافي بدون الاعضاء المأكولة لثلاث سلالات من فروج اللحم (لومان وروز وهبرد)

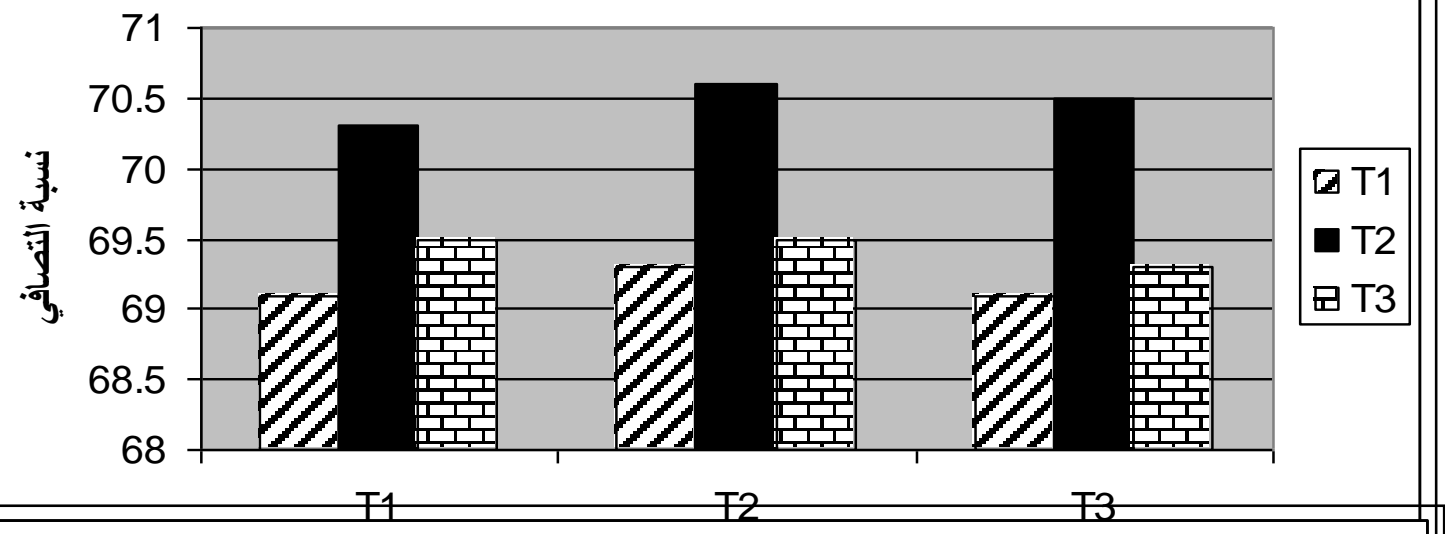

الثكل 2 تأثير اضافة تركيزين مختلفين من الكروم العضوي في نسب التصافي مع

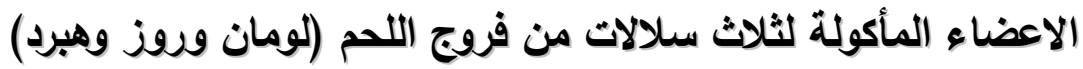

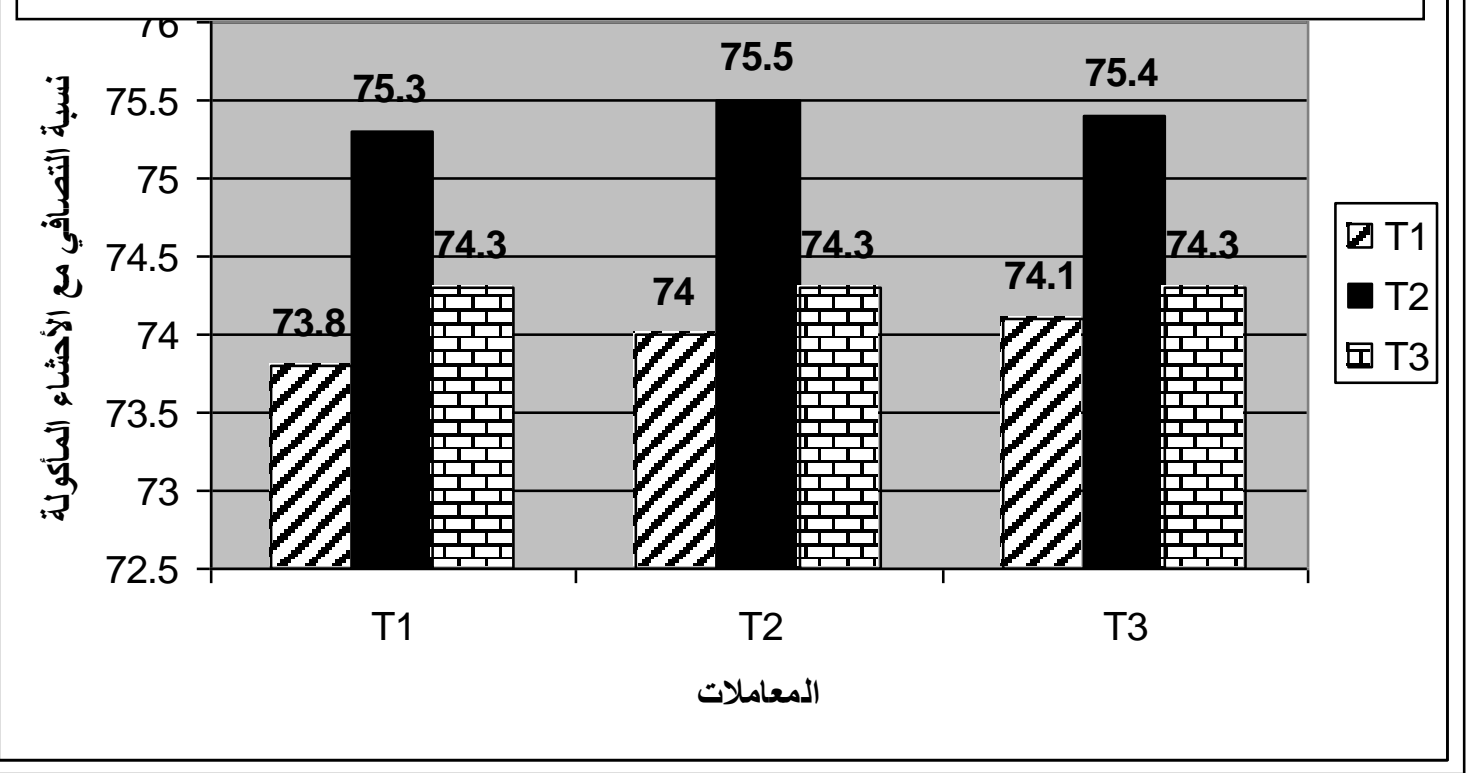


المجلة الطبية البيطرية العراقية، المجلد 30، العدد 2، السنة 2006

الجدول 1 تأثير اضافة تركيزين مختلفين من الكروم العضوي في نسب اوزلن الاعضاء المأكولة لثلاث سلالات من فروج اللحم (لومان وروز وهبرد):

\begin{tabular}{|c|c|c|c|}
\hline القانصة \% & الكبد \% \% & القلب \% & المعاملات \\
\hline 2.16 & 2.11 & 0.40 & T1Cr0 \\
\hline 2.34 & 2.25 & 0.43 & T1Cr1 \\
\hline 2.29 & 2.00 & 0.49 & T1Cr2 \\
\hline 2.30 & 1.97 & 0.40 & T2Cr0 \\
\hline 2.40 & 2.00 & 0.47 & T2Cr1 \\
\hline 2.20 & 2.20 & 0.40 & $\mathrm{~T} 2 \mathrm{Cr} 2$ \\
\hline 2.35 & 2.23 & 0.45 & T3Cr0 \\
\hline 2.4 & 2.00 & 0.46 & T3Cr1 \\
\hline 2.30 & 2.40 & 0.42 & T3Cr2 \\
\hline N.S & N.S & N.S & الهجن \\
\hline N.S & N.S & N.S & تركيز الكروم \\
\hline N.S & N.S & N.S & الهجن X الكروم \\
\hline
\end{tabular}

التحليل الكيميائي للحم :

يتضح من الجدول 3 ظهور اختلافات معنوية في معدلات قيم نسب الرطوبة والبروثين والدهن

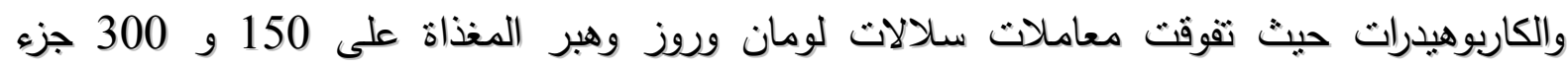

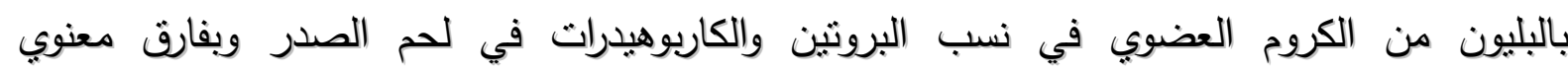
عن (P<0.05)

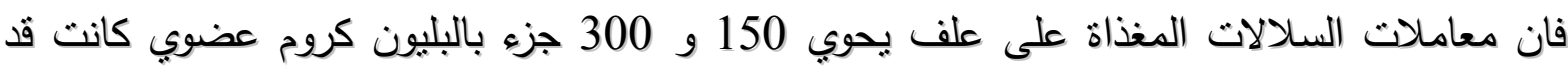

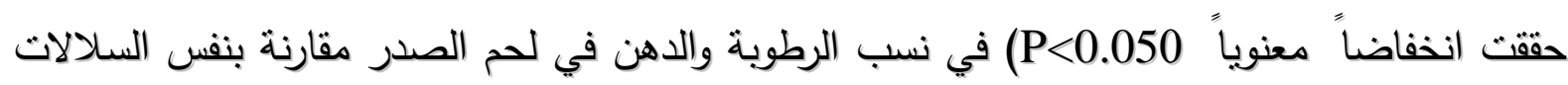

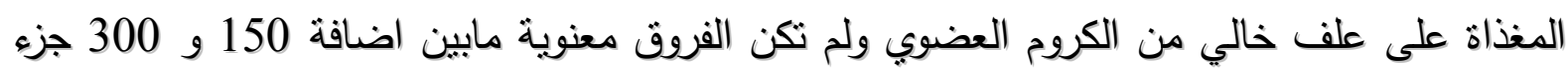

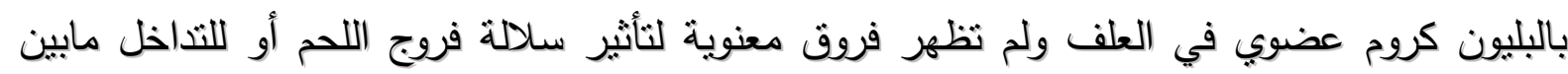

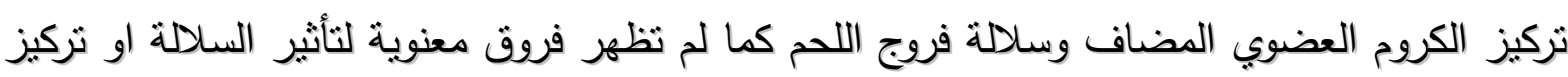

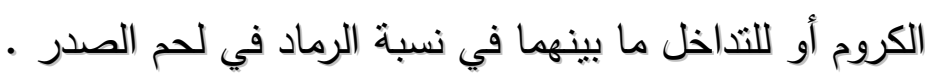

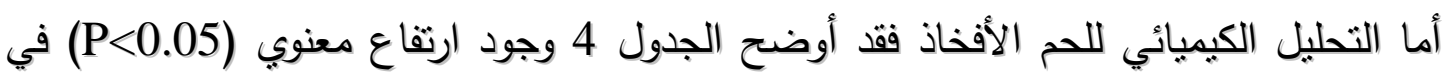
نسب البروثين والكاربوهيدرات والرطوبة في لحم أفخاذ سلالات لومان وروز وهبرد المغذاة على علف 
يحوي 150 و 300 جزء بالبليون كروم عضوي مقارنة بمعاملات نفس السلالات المغذاة على علف

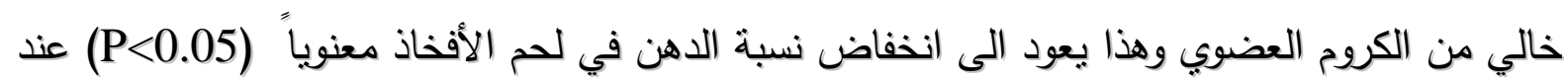
التغذية على الكروم العضوي من لكروم لعضوي وهن

الجدول 2 تأثير اضافة تركيزين مختلفين من الكروم العضوي في نسب اونان قطيعات ذبيحة ثلاث سلالات من فروج اللحم (لومان وروز وهبرد):

\begin{tabular}{|c|c|c|c|c|c|}
\hline رقبة ر ر & ظهر \% & أجنحة \% & فذذ \% & صدر \% & $\begin{array}{c}\text { القطعيات } \\
\text { المعالمعاملات }\end{array}$ \\
\hline $5.59^{\mathrm{cd}}$ & $25.13^{\mathrm{a}}$ & $12.45^{\mathrm{ab}}$ & $31.96^{\mathrm{a}}$ & $27.87^{b}$ & T1Cr0 \\
\hline $5.53^{\mathrm{cd}}$ & $22.55^{\mathrm{bc}}$ & $11.62^{b}$ & $31.45^{\mathrm{ab}}$ & $28.85^{\mathrm{a}}$ & T1Cr1 \\
\hline $5.43^{\mathrm{d}}$ & $22.09^{\mathrm{c}}$ & $13.45^{\mathrm{a}}$ & $30.88^{\mathrm{ab}}$ & $28.15^{\mathrm{a}}$ & T1Cr2 \\
\hline $5.74 b^{c d}$ & $22.72^{\mathrm{bc}}$ & $12.18^{\mathrm{ab}}$ & $32.23^{\mathrm{a}}$ & $27.13^{b}$ & T2Cr0 \\
\hline $5.53^{\mathrm{cd}}$ & $23.21^{a b c}$ & $12.32^{\mathrm{ab}}$ & $30.45^{\mathrm{b}}$ & $28.49^{\mathrm{a}}$ & T2Cr1 \\
\hline $6.29^{\mathrm{ab}}$ & $22.29^{\mathrm{c}}$ & $13.01^{\mathrm{ab}}$ & $30.63^{b}$ & $27.78^{\mathrm{ab}}$ & T2Cr2 \\
\hline $6.70^{\mathrm{a}}$ & $24.10^{a b c}$ & $11.51^{b}$ & $31.89^{\mathrm{a}}$ & $25.80^{\mathrm{c}}$ & T3Cr0 \\
\hline $6.18^{\mathrm{abc}}$ & $25.46^{\mathrm{a}}$ & $11.78^{b}$ & $31.20^{\mathrm{ab}}$ & $25.38^{\mathrm{c}}$ & T3Cr1 \\
\hline $6.32^{\mathrm{ab}}$ & $24.79^{\mathrm{ab}}$ & $11.68^{b}$ & $29.97^{b}$ & $27.24^{b}$ & T3Cr2 \\
\hline$*$ & $*$ & $*$ & N.S & * & الهجن \\
\hline N.S & N.S & N.S & $*$ & $*$ & تركيز الكروم \\
\hline$*$ & $*$ & $*$ & N.S & $*$ & الهجن X التركيز \\
\hline
\end{tabular}


المجلة الطبية البيطرية العراقية، المجلد 30، العدد 2، السنة 2006

الجدول 3 تأثير اضافة تركيزين مختلفين من الكروم العضوي في التحليل الكيميائي للحم صدر ثلاث سلالات من فروج اللحم (لومان وروز وهبرد) :

\begin{tabular}{|c|c|c|c|c|c|}
\hline الكاريوهيدرات \% & الدهن \% & البروتين \% & الرماد \% & الرطوية \% & المعاملات \\
\hline $0.67^{b}$ & $3.33^{\mathrm{a}}$ & $21.00^{b}$ & 0.86 & $74.14^{\mathrm{a}}$ & T1Cro. \\
\hline $0.93^{\mathrm{a}}$ & $2.40^{\mathrm{b}}$ & $21.73^{\mathrm{a}}$ & 0.86 & $73.08^{b}$ & $\mathrm{~T}^{1 C r_{1}}$ \\
\hline $0.83^{\mathrm{a}}$ & $2.75^{b}$ & $22.50^{\mathrm{a}}$ & 0.86 & $73.06^{b}$ & $\mathrm{~T} \mathrm{Cr}_{2}$ \\
\hline $0.69^{b}$ & $3.30^{\mathrm{a}}$ & $21.20^{\mathrm{b}}$ & 0.84 & $73.97^{\mathrm{a}}$ & $\mathrm{T}^{2} \mathrm{Cr}_{0}$ \\
\hline $0.96^{\mathrm{a}}$ & $2.50^{\mathrm{b}}$ & $22.50^{\mathrm{a}}$ & 0.85 & $73.19^{b}$ & $\mathrm{~T}_{2} \mathrm{Cr}_{1}$ \\
\hline $0.90^{\mathrm{a}}$ & $2.66^{b}$ & $22.43^{\mathrm{a}}$ & 0.85 & $73.16^{b}$ & $\mathrm{~T}_{2} \mathrm{Cr}_{2}$ \\
\hline $0.70^{b}$ & $3.53^{\mathrm{a}}$ & $22.03^{b}$ & 0.85 & $73.89^{\mathrm{a}}$ & $\mathrm{T} \mathrm{Cr}_{0}$ \\
\hline $0.85^{\mathrm{a}}$ & $2.55^{\mathrm{b}}$ & $22.73^{\mathrm{a}}$ & 0.87 & $73.88^{b}$ & T3Cr $_{1}$ \\
\hline $1.00^{\mathrm{a}}$ & $2.96^{b}$ & $22.00^{\mathrm{a}}$ & 0.86 & $73.18^{b}$ & $\mathrm{~T}_{3} \mathrm{Cr}_{2}$ \\
\hline N.S & N.S & N.S & N.S & N.S & السلالة \\
\hline$*$ & $*$ & $*$ & N.S & $*$ & التركيز \\
\hline N.S & N.S & N.S & N.S & N.S & الهجن X \\
\hline
\end{tabular}

الأحرف الصغيرة المختلفة ضمن العمود الواحد تشير الى وجود فروق معنوية .

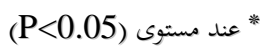
ع.S 
المجلة الطبية البيطرية العراقية، المجلد 30، العدد 2، السنة 2006

الجدول 4 تأثير اضافة تركيزين مختلفين من الكروم العضوي في التحليل الكيميائي للحم أفخاذ ثلاث سلالات من فروج اللحم (لومان وروز وهبرد) :

\begin{tabular}{|c|c|c|c|c|c|}
\hline الكاريوهيدرات \% & الدهن \% & البروتين \% & الرماد \% & الرطوية \% & المعاملات \\
\hline $0.53^{b}$ & $9.72^{\mathrm{a}}$ & $19.40^{\mathrm{b}}$ & 0.73 & $69.62^{b}$ & T1Cro. \\
\hline $0.86^{\mathrm{a}}$ & $8.49^{b}$ & $20.00^{\mathrm{a}}$ & 0.72 & $69.93^{\mathrm{a}}$ & $\mathrm{T1Cr}_{1}$ \\
\hline $0.81^{\mathrm{a}}$ & $8.21^{b}$ & $20.13^{a}$ & 0.74 & $70.11^{\mathrm{a}}$ & $\mathrm{T1Cr}_{2}$ \\
\hline $0.49^{b}$ & $9.80^{\mathrm{a}}$ & $19.20^{\mathrm{b}}$ & 0.72 & $69.79^{b}$ & $\mathbf{T 2 C r}_{0}$ \\
\hline $0.85^{\mathrm{a}}$ & $8.37^{b}$ & $19.73^{\mathrm{a}}$ & 0.72 & $70.33^{\mathrm{a}}$ & $\mathbf{T}_{2} \mathrm{Cr}_{1}$ \\
\hline $0.86^{\mathrm{a}}$ & $8.30^{b}$ & $20.03^{\mathrm{a}}$ & 0.73 & $70.08^{\mathrm{a}}$ & $\mathbf{T}_{2} \mathrm{Cr}_{2}$ \\
\hline $0.50^{\mathrm{b}}$ & $9.70^{\mathrm{a}}$ & $19.30^{\mathrm{b}}$ & 0.73 & $69.77^{b}$ & $\mathbf{T 3 C r}_{0}$ \\
\hline $0.83^{\mathrm{a}}$ & $8.40^{\mathrm{b}}$ & $20.13^{\mathrm{a}}$ & 0.74 & $69.90^{\mathrm{a}}$ & $\mathrm{T} \mathrm{Cr}_{1}$ \\
\hline $0.82^{\mathrm{a}}$ & $8.47^{b}$ & $20.20^{\mathrm{a}}$ & 0.73 & $69.90^{\mathrm{a}}$ & $\mathrm{T3Cr}_{2}$ \\
\hline N.S & N.S & N.S & N.S & N.S & السلالة \\
\hline$*$ & $*$ & $*$ & N.S & $*$ & التركيز \\
\hline N.S & N.S & N.S & N.S & N.S & التركيز \\
\hline
\end{tabular}

الأحرف الصغيرة المختلفة ضمن العمود الواحد تشير الى وجود فروق معنوية .

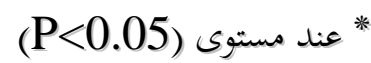
ع.S

\section{المناقشة}

أن ارتفاع نسبة التصافي في معاملات سلالات فروج اللحم لومان وروز وهبرد المغذاة

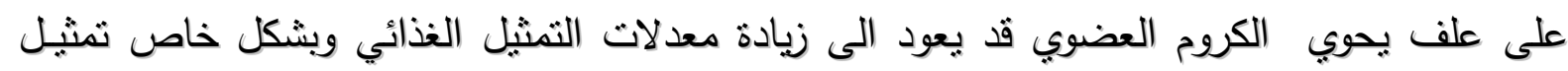

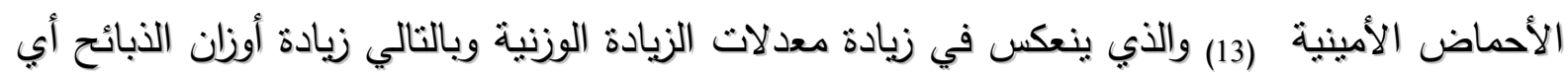

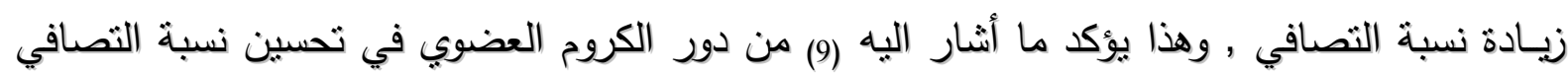

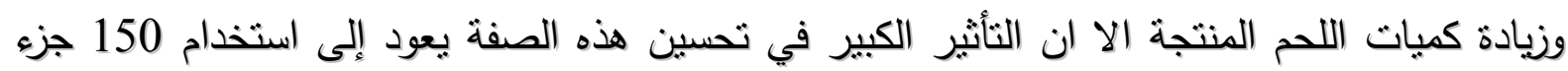

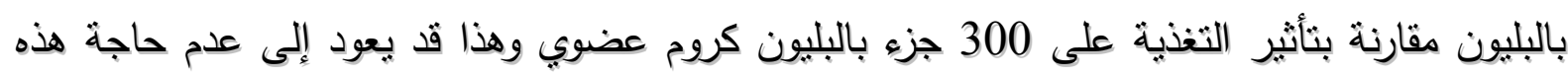

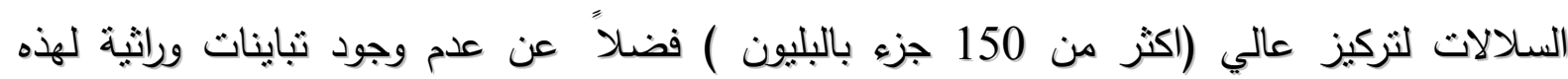


السلالات الثلاث في نسبة التصافي , وعلى الرغم من وجود ثباينات وراثية مابين الانواع والسلالات في

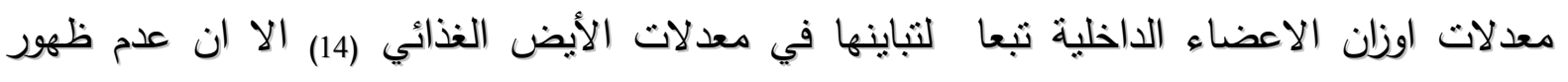

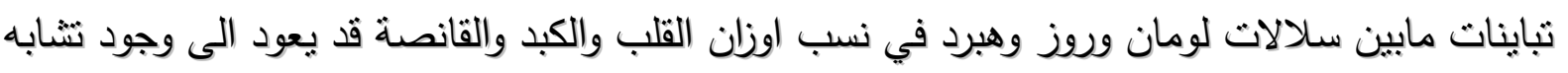

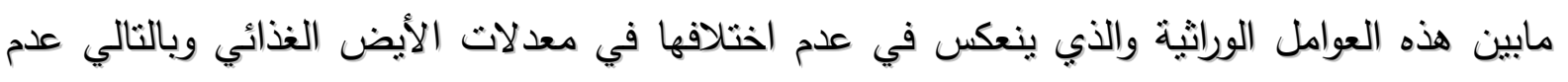

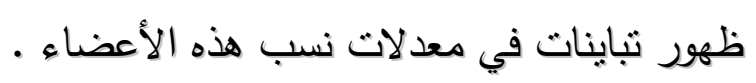

قطعتي الصدر والافخاذ من القطيعات الرئيسية في ذبائح الدجاج (15) لإحنوائها على الاعلى

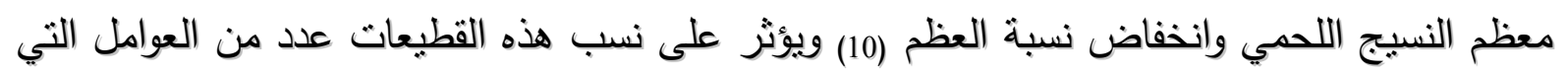
تأتي في مقدمتها العوامل الوراثية حيث نتباين نسب هذه القطيعات ونسبة ماتحنويه من اللحم والعظم

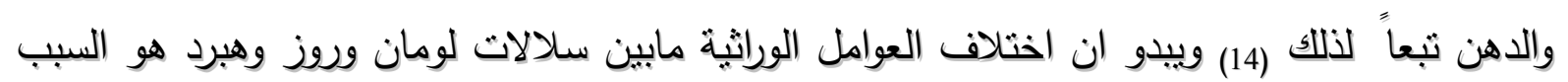

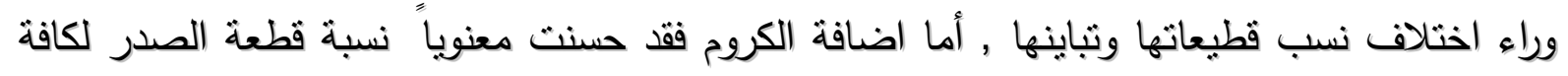

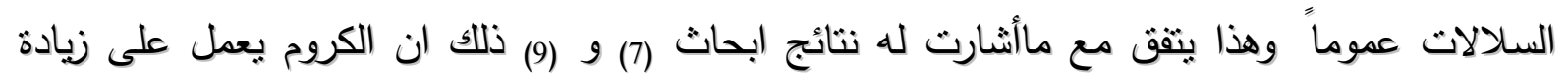

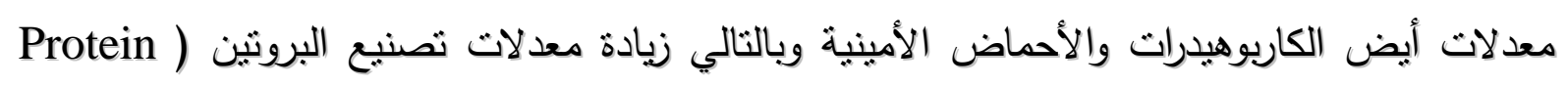
(Synthesis

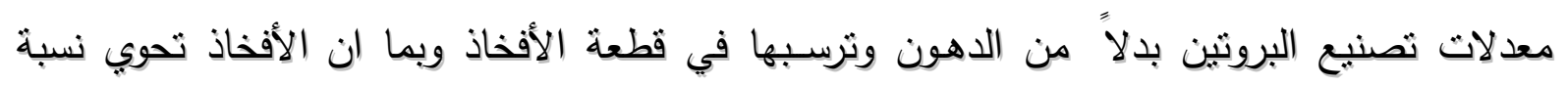

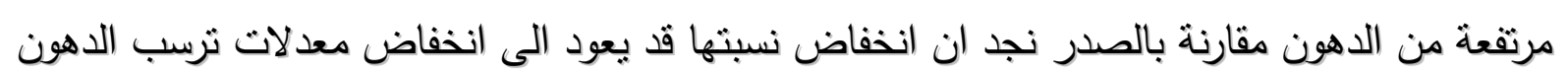
فيها .

التحليل الكيميائي للدم الصدر والأفخاذ أوضح وجود ارتفاع معنوي في نسب البروتين

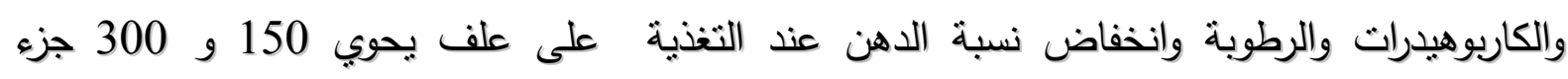

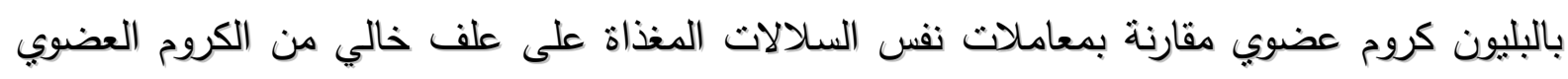

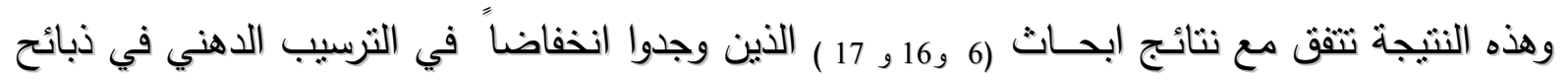

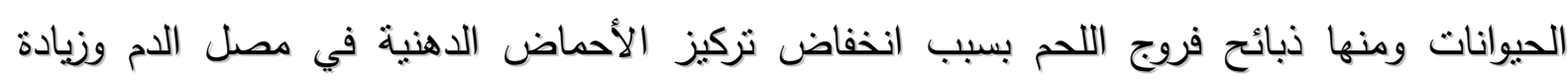

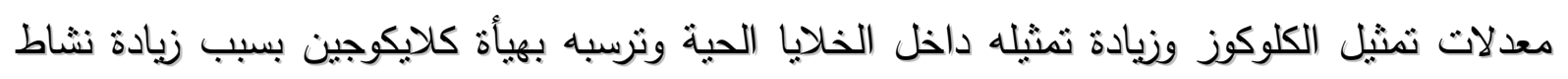

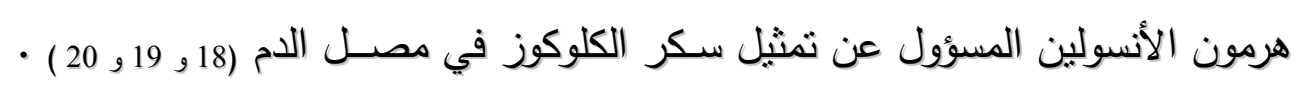
المصادر

\section{Flodin,N.W.1988.Pharmacology of micro nutrition , 14 chromium, (N.W.flodin,Ed.) $20.247-254$.}

2. Mordenti,A.A.piva. and G.Piva .1997.The European prespective on organic chromium in animal nutrition .Pages 227-240.in Biotechnology in the feed 
industry proc.Alltech's thirteenth Ann.Symp.T.P.lyons and K.A. Jacques .Nottingham University Press .nottingham.U.K.

3. Mordenti,A.A.piva. and G.Piva .1997.The European prespective on organic chromium in animal nutrition .Pages 227-240.in Biotechnology in the feed industry proc.Alltech's thirteenth Ann.Symp.T.P.lyons and K.A. Jacques .Nottingham University Press .nottingham.U.K.

4. Vandelight,C.P.A.,M.D..Lindemann, and $\quad$ G.L.Cromwell 2002. Assessment of Chromium tripicolinate supplementation and Dietary energy level and source on growth ,carcass and blood citeria in growing pigs J.Anim.Sci.80:483-493.(Abstract/free Full Text).

5. الثديدي ،شهزاد محمد جعفر وغالب علوان القيسي وزينب عبد الزهزة .2005 ـ مقارنة استخدام

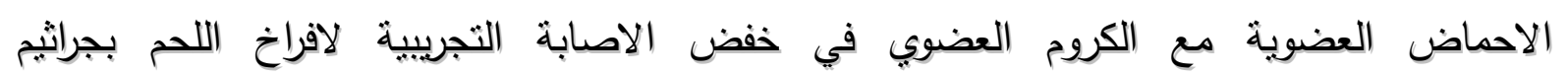

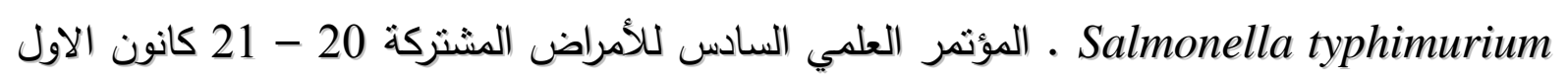
2005 / كلية الطب البيطري / جامعة بغداد .

6. Ward,T.L., and Southern L.L. 1995.Effect of dietary chromium source on growth carcass characteristies, and plasma Metabole and hormone concentration in growing - finishing swine . J.Anim Sci.73(Suppl.):189.

7. Hossain ,S.M.1995 . Effect of chromium yeast on performance and carcass quality of broiler. Alltech's Elerenth Ann.Symp. Poster.Presentation.

8. Savoinin,G.C.,Sgoifo Rossi,D.Cevolani,F.polidori and Deforto V.1996. Utilization Di Lievito coltivato Su Sabstrato contenente Cromonella dieta del suino pesante.Riv.Suinoecoltura.37:145-149.

9. Hossain , S . M . ; Barreto , S.L and Silva , C.G . 1997 . Growth performance and carcass composition of broiler fed supplemental chromium from chromium yeast. Animal feed Science and Technology.

10. الفياض ، حمدي عبد العزيز وسعد عبد الحسين ناجي . 1989 ـ تكنولوجيا منتجات الدواجن ط1

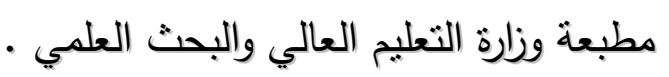

11. AOAC,1980.Offical Methods for Analysis of Association officals of analytical chemists. $11^{\text {th }}$ ed .Washington.D.C.

12. SAS.2001.SAS/TAT user's guide, version $7.4^{\text {th }}$ ed. SAS Institute Inc.

13. Gary,N.C.

14. Evans,G.W.and Bowman,T.D.1992.Chromium picolinate Increases membrane fluidity and rate of insulin Internalization .J.Inorg .Biochem.26:243.

$$
\begin{aligned}
& \text { 15. الفياض ، حمدي عبد العزيز وفارس عبد علي العبدي ونجم اسماعيل الحديثي ويشرى سعدي }
\end{aligned}
$$

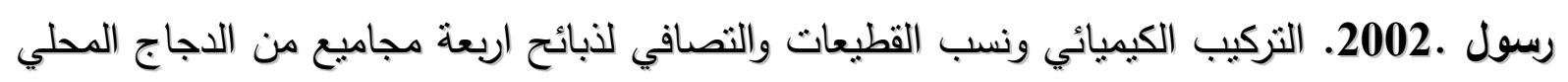

$$
\begin{aligned}
& \text { ـ مجلة إباء للأبحاث الزراعية 12: 125- } 134 \text {. } 13 \text {. }
\end{aligned}
$$


16. Mountney, G.L. 1976. Poultry Products Technology. $2^{\text {nd }}$ ed. The AVI Publishing Company . Westport, Connecticut.USA.

17. Ward,T.L., Southern L.L.and S . L . Boleman.1993 . Effect of dietary Chromium picolinate on growth, nitrogen balance and body Composition of growing broiler chicks poult.Sci.72(Suppl.1) :37.

18. Page,T.G.,L.L..Soathern,T.L.Ward and D.L.thompson and Serum and D.L.Thompson,Jr.1993.Effect of chromium picolinata on growth and serum and carcass traits of growing finishing pigs .J.Anim.Sci.656-662.

19. Brown , R.O.;Foriones-lynn,S.;Cross,R.E. and Heizer,W.D.1986.

Chromium efficiency after long - term total parenteral nutrition .Dig. Dis .and Sci.31:661.

20. Mertz,W.1987.Interaction of chromium with insulin :A progress report.Nutr.Rev.56:174-177.

21. Guan ,X.F .; Snow ,J.L .;Ku ,P.;Burtor,J. and Trotteir,N.L.1997.Effect of Dietary chromium supplementation on plasma glucose kineties in borrows and gitts. J. Anim .Sei. 75.(Suppl,1):189. 